\title{
Finite element analysis of rapid canine retraction through reducing resistance and distraction
}

\author{
Junjie XUE ${ }^{1,2}$, Niansong YE 1 , Xin YANG ${ }^{1}$, Sheng WANG ${ }^{1}$, Jing WANG ${ }^{3}$, Yan WANG ${ }^{1}$, Jingyu LI ${ }^{1}$, Congbo MI², Wenli LAI \\ 1- State Key Laboratory of Oral Disease, Department of Orthodontics, West China Hospital of Stomatology, Sichuan University, Chengdu, Sichuan, P.R. China. \\ 2- Department of Orthodontics, First Affiliated Hospital of Xinjiang Medical University, Urumqi. Xinjiang, P.R. China. \\ 3- Department of Stomatology, Shanghai Tenth People's Hospital, Tongji University School of Medicine, Shanghai, P.R. China.
}

Corresponding address: Wenli Lai - Department of Orthodontics - State Key Laboratory of Oral Diseases - West China Hospital of Stomatology - Sichuan University - Address: No.14, $3^{\text {rd }}$ Sec. - Renminnan Rd. - Chengdu - Sichuan - China - Zip code: 610041 - Phone: 86-028-85501442 - e-mail: wenlilai@hotmail.com

Submitted: June 7, 2013 - Modification: August 20, 2013 - Accepted: September 17, 2013

\section{ABSTRACT}

\begin{abstract}
$\mathrm{O}$ bjective: The aims of this study were to compare different surgical approaches to rapid canine retraction by designing and selecting the most effective method of reducing resistance by a three-dimensional finite element analysis. Material and Methods: Threedimensional finite element models of different approaches to rapid canine retraction by reducing resistance and distraction were established, including maxillary teeth, periodontal ligament, and alveolar. The models were designed to dissect the periodontal ligament, root, and alveolar separately. A $1.5 \mathrm{~N}$ force vector was loaded bilaterally to the center of the crown between first molar and canine, to retract the canine distally. The value of total deformation was used to assess the initial displacement of the canine and molar at the beginning of force loading. Stress intensity and force distribution were analyzed and evaluated by Ansys 13.0 through comparison of equivalent (von Mises) stress and maximum shear stress. Results: The maximum value of total deformation with the three kinds of models occurred in the distal part of the canine crown and gradually reduced from the crown to the apex of the canine; compared with the canines in model 3 and model 1 , the canine in model 2 had the maximum value of displacement, up to $1.9812 \mathrm{~mm}$. The lowest equivalent (von Mises) stress and the lowest maximum shear stress were concentrated mainly on the distal side of the canine root in model 2 . The distribution of equivalent (von Mises) stress and maximum shear stress on the PDL of the canine in the three models was highly concentrated on the distal edge of the canine cervix. Conclusions: Removal of the bone in the pathway of canine retraction results in low stress intensity for canine movement. Periodontal distraction aided by surgical undermining of the interseptal bone would reduce resistance and effectively accelerate the speed of canine retraction.
\end{abstract}

Keywords: Distraction osteogenesis. Oral surgical procedures. Orthodontics. Finite element analysis.

\section{INTRODUCTION}

Currently, conventional fixed orthodontic treatment requires about 1-2 years ${ }^{4}$. More time is required for extraction cases, such as for adult patients, which is a great concern and poses high risk of caries ${ }^{5}$, external root resorption ${ }^{18}$. Thus, accelerating orthodontic tooth movement and the resulting shortening of the treatment duration would be beneficial. Many researchers have utilized different biochemical methods involving medications to improve the speed and quality of orthodontic treatment, but the systemic influence on the body's metabolism makes this difficult to apply in Orthodontics. Recently, investigators have begun studying local techniques for stimulating better orthodontic tooth movement. Surgically aided rapid tooth movement has become one of the novel techniques for accelerating canine retraction.

For premolar extraction cases ${ }^{26}$, the first phase of treatment is distal movement of the canines. Biologic tooth movement can be achieved ${ }^{23}$ with conventional orthodontic treatment techniques, but the canine retraction phase usually lasts for 6 to 8 months. Extraoral or intraoral anchorage 
mechanics are required to maintain the space obtained during canine distalization, particularly when maximum anchorage is required. To date, several novel surgical modalities and techniques for rapid canine retraction have been reported to accelerate canine distalization. These attempts fall mainly into two categories: the first, dentoalveolar distraction osteogenesis, evolved from distraction osteogenesis $2,8,16$. Osteotomies surrounding the canines are created to achieve rapid movement of the canines in the dentoalveolar segment, in compliance with the principles of distraction osteogenesis. The second technique is periodontal distraction aided by surgical undermining of the interseptal bone ${ }^{14,20}$. Nevertheless, the question remains regarding which of the above techniques is more effective for rapid canine retraction.

Until now, these research modalities and techniques for accelerating canine retraction have been applied in animal experiments and clinical case reports. However, the biomechanics of the application process has not been studied.

Biomechanics is crucial in all aspects of Dentistry, especially in Orthodontics. After the application of an inappropriate external force to the oral environment, the development of a stress field in the supporting tissues typically leads to an unsuccessful outcome. Finite element analysis has become a powerful tool for dental biomechanical research due to its increased availability, capacity, and ease of use of computer software in biologic modeling. It can be considerably effective and, most importantly, non-invasive.

What is the status of stress in the maxillary teeth, the periodontal ligament (PDL), and alveolar bone during rapid canine retraction? How does the biomechanics differ between dentoalveolar distraction osteogenesis and periodontal distraction aided by surgical undermining of the interseptal bone? Will it produce side-effects in addition to tooth movement? To address these questions via the finite element (FE) method, in this study we constructed a three-dimensional (3D) finite element model of the maxillary teeth, the periodontal ligament (PDL), and alveolar bone after extracting the first premolars.

\section{MATERIAL AND METHODS}

\section{D FE model generation}

For better simulation of the movement of canine retraction during orthodontic treatment with different auxiliary surgical methods, a patient with bimaxillary protrusion was selected from the orthodontic department of First Affiliated Hospital of Xinjiang Medical University. The patient was informed of the experimental protocols and provided informed consent. The study protocol and patient consents were approved by the Ethics Review Committee. Four maxillary first premolars were extracted as part of the treatment.

After the maxillary teeth were aligned and leveled by means of an invisible aligner, images of the patient's teeth and alveolar bone were obtained in a DICOM (digital imaging and communication in medicine) data format via 64-slice (a slice thickness of $0.5 \mathrm{~mm}$ ) spiral computed tomography (CT). The geometric shapes of the maxillary teeth and alveolar bone (cancellous and cortical bone) were reconstructed by MIMICS (version 15.0; Materialise, Leuven, Belgium), then exported to Geomagic Studio (2013, Raindrop Inc., Rock Hill, South Carolina, U.S.A.), and modified by Unigraphics NX (version 8.5, Siemens, Münich, Germany). The initial 3D finite element model of the maxilla and the maxillary teeth was established with 416,931 nodes and 227,784 solid elements (Figure 1 ), and analyzed by ANSYS Workbench (version 13.0;

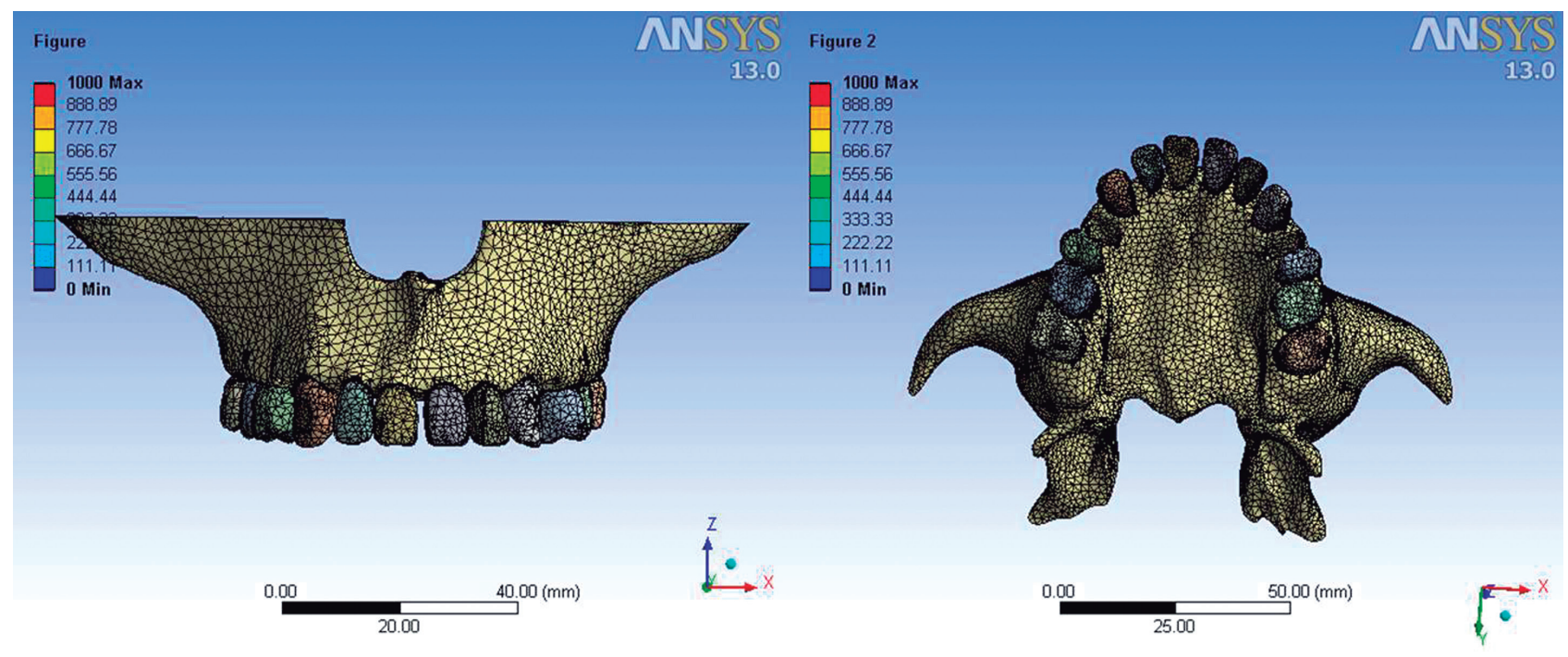

Figure 1- The initial 3D finite element model of the maxilla and the maxillary teeth 
ANSYS Inc., Canonsburg, Pennsylvania, U.S.A.). Although periodontal ligament thicknesses differ according to age, position, and individual variations, the thickness of the periodontal ligament was considered to be consistently $0.25 \mathrm{~mm}^{3,21}$. The 3D finite element models of the alveolar bone were fabricated to fit the teeth and the periodontal ligament, and the thickness of cortical bone was considered to be $2 \mathrm{~mm}^{13,17}$.

In this study, we defined the non-surgicallyaided canine retraction model as model 1 . The numbers of nodes and elements of the initial model are shown in Figure 2. According to the two types of auxiliary surgical methods for accelerating canine retraction, we simulated the same surgical process in the initial 3D finite element model, and two 3D FEMs were generated based on the two different surgeries for comparison with the initial model (model 1).

We defined the FEM of periodontal distraction aided by surgical undermining of the interseptal bone as model 2 (PD). After the first premolar was removed, the interseptal bone mesial to the extraction socket was undermined. The bone in the premolar socket was eliminated vertically along the buccal and lingual sides, extending obliquely toward the base of the interseptal bone to weaken resistance. The interseptal bone was not cut

\begin{tabular}{|c|c|c|}
\hline Models & Number of nodes & Number of elements \\
\hline Cancellous bone & 69.897 & 41.814 \\
\hline Cortical bone & 118.762 & 68.648 \\
\hline Canine & 7.293 & 4.181 \\
\hline Second premolar & 3.933 & 2.241 \\
\hline First molar & 5.961 & 3.497 \\
\hline PDL of canine & 17.383 & 8.584 \\
\hline PDL of second premolar & 11.482 & 5.645 \\
\hline PDL of first molar & 18.873 & 9.306 \\
\hline
\end{tabular}

Figure 2- Number of nodes and elements generated for initial model $\mathrm{PDL}=$ periodontal ligament

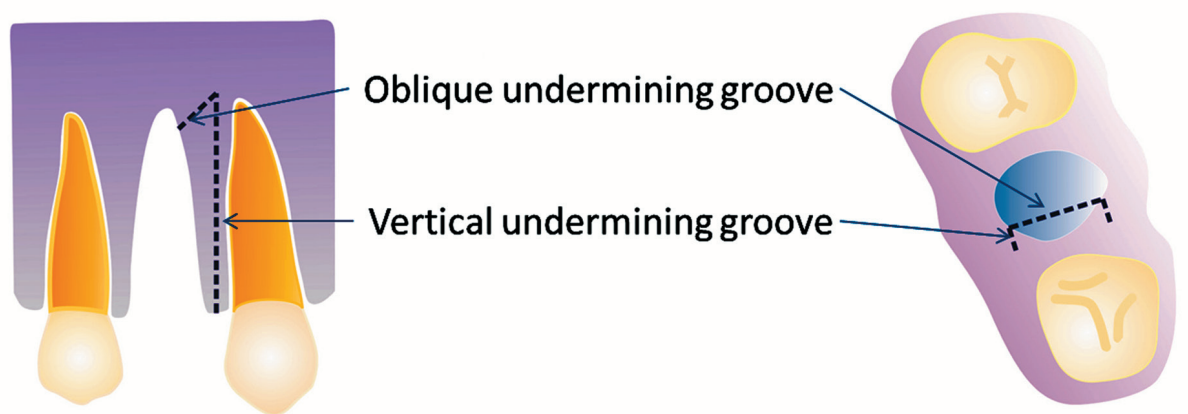

Figure 3- Surgical techniques for undermining interseptal bone distal to the canine. No cuts are performed on buccal and lingual plates. Note depths and positions of undermining grooves

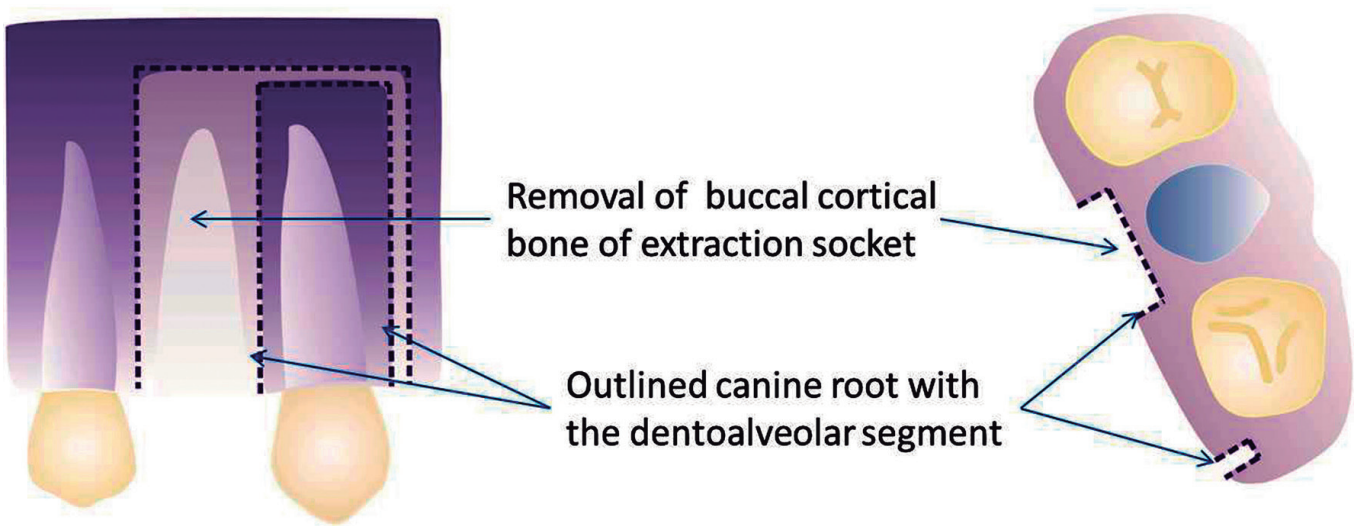

Figure 4- Surgical techniques for dentoalveolar distraction osteogenesis 
through mesio-distally toward the canine (Figure 3 ). The depth of the undermining grooves was dependent on the thickness of the interseptal bone.

The final model (model 3) was the FEM model of dentoalveolar distraction osteogenesis (DDO). The first premolar was extracted, and the buccal cortical bone of the extraction socket was removed. An osteotomy line was determined between the buccal root apex of the first premolar and canine apex (Figure 4).The root of the upper canine tooth was outlined mesially and distally, with the dentoalveolar segment to be used as a transport disc at the apical region. The cortical bone, especially at the level of the apical region, was also eliminated for maximal bodily movement during distraction.

\section{Material properties}

The mechanical properties of the teeth, PDL, and cortical and cancellous bone were defined as isotropic, homogenous, and linearly elastic. Young's modulus and Poisson's ratio for all materials were calculated according to previously described methods (Figure 5) 19,24 . The boundary condition was defined as the exterior border of the maxilla.

\begin{tabular}{|c|c|c|}
\hline Material & Young's Modulus (MPa) & Poisson's Ratio \\
\hline Cancellous bone & 1.370 & 0.3 \\
\hline Cortical bone & 13.700 & 0.3 \\
\hline Tooth & 20.000 & 0.3 \\
\hline PDL & 0.68 & 0.3 \\
\hline
\end{tabular}

Figure 5- Mechanical properties of the materials used in the finite element models PDL=periodontal ligament

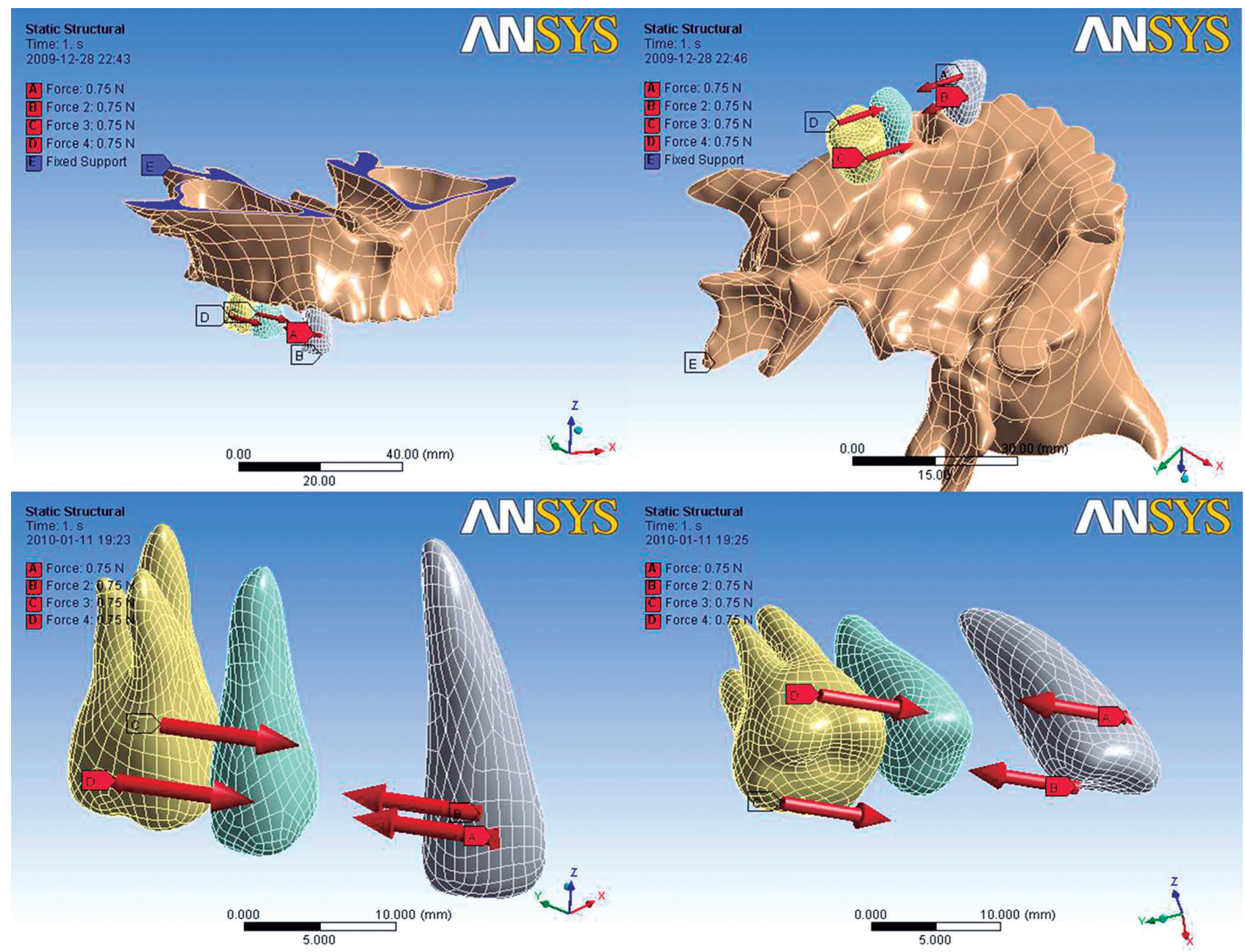

Figure 6- The coordinate system. A mesiodistal simulated retraction force of $1.5 \mathrm{~N}$ was loaded bilaterally to the center of the crown between the first molar and the canine 


\section{Experimental conditions}

The coordinate system and directions of forces and loads are shown in Figure 6. A simulated retraction force of $1.5 \mathrm{~N}$ (approximately $150 \mathrm{~g}$ ) was loaded bilaterally to the center of the crown between the first molar and the canine, and stress distribution and its magnitude were analyzed by ANSYS Workbench, a 3D finite element analysis program. An assessment of the stress on the bone elements was performed by von Mises equivalent stress and maximum shear stress, and we used the total deformation value to assess the initial displacement of the teeth and the PDL.

\section{RESULTS}

\section{Canine}

Immediately after loading of the retraction forces of $1.5 \mathrm{~N}$ (approximately $150 \mathrm{~g}$ ) bilaterally between the crown of the first molar and the canine, the initial displacement of the canine was highly concentrated in the distal area of the crown in all three models. Compared with the total deformation of the canine in model 1 (non-surgical) and model 3 (DDO), the canine in model 2 had the maximum value of total deformation of $1.9812 \mathrm{~mm}$ (Table 1 , Figure 7).

Compared with the maximum value of equivalent

Table 1- Total Deformation values induced in three models

\begin{tabular}{lcccc}
\hline & \multicolumn{3}{c}{ Total Deformation values (mm) } & PDL of First molar \\
\hline Non-surgical & Canine & First molar & PDL of Canine & PD \\
PD & 1.8925 & 0.15441 & 0.10237 & 0.070115 \\
DDO & 1.9812 & 0.12674 & 0.13265 & 0.069375 \\
\hline
\end{tabular}

$\mathrm{PD}=$ periodontal distraction; $\mathrm{PDL}=$ periodontal ligament

$\mathrm{DDO}=$ dentoalveolar distraction osteogenesis

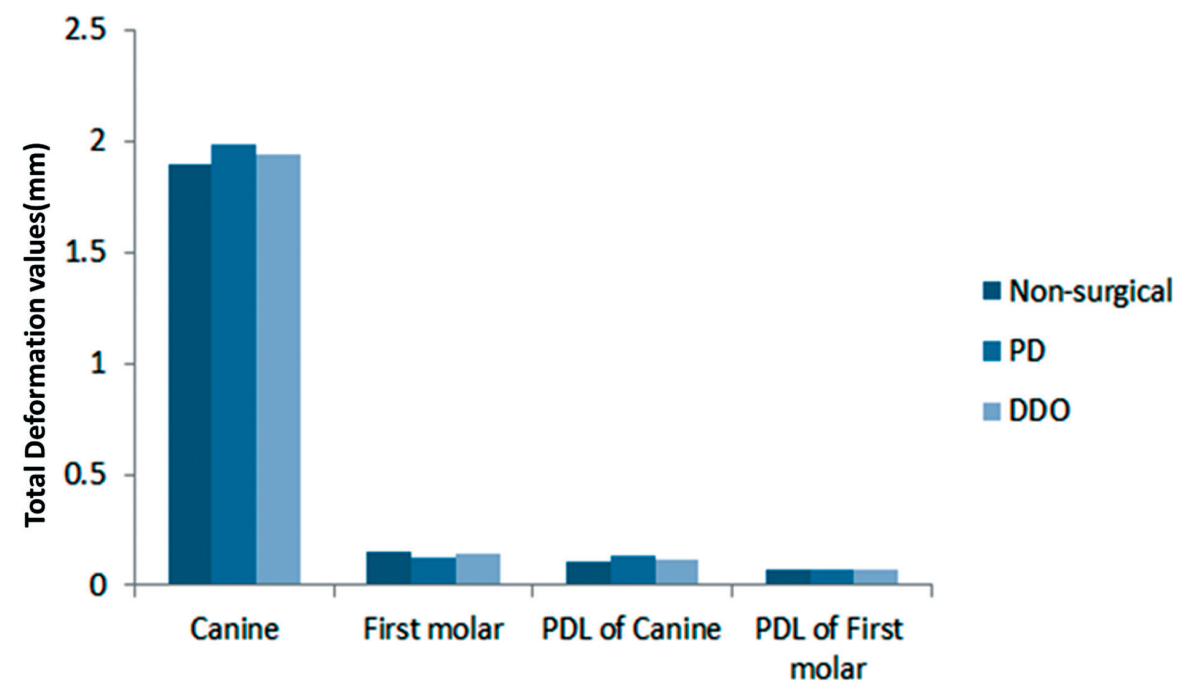

Figure 7- Total deformation values induced in different parts of three models. PDL= periodontal ligament

Table 2- Equivalent (von Mises) Stress values induced in three models

\begin{tabular}{cccccc}
\hline & \multicolumn{3}{c}{ Maximum Von Mises stress values (MPa) } & & \\
& Canine & First molar & PDL of Canine & $\begin{array}{c}\text { PDL of First } \\
\text { molar }\end{array}$ & Cortical bone \\
\hline Non-surgical & 0.56251 & 0.57389 & 0.049053 & 0.035727 & 1,7942 \\
PD & 0.35494 & 0.43203 & 0.043629 & 0.03509 & 0.622 \\
DDO & 0.45463 & 0.45208 & 0.043347 & 0.035098 & 0.6796 \\
\hline
\end{tabular}

$\mathrm{PD}=$ periodontal distraction; $\mathrm{PDL}=$ periodontal ligament

$\mathrm{DDO}=$ dentoalveolar distraction osteogenesis 
(von Mises) stress on the canine in models 1 and 3 (DDO), the canine in model 2 (PD) had the lowest equivalent (von Mises) stress value of $0.35494 \mathrm{MPa}$ (Table 2, Figure 8). Likewise, compared with model 1 (non-surgical) and model 3 (DDO), the value of maximum shear stress of the canine in model 2 (PD) was the lowest (Table 3).

\section{Molar}

As the anchor tooth, the value of total deformation on the first molar in model 2 was $0.12674 \mathrm{~mm}$, which is the lowest in comparison with that of the molar in model 1 (non-surgical) and model 3 (DDO) (Table 1).

Distribution of maximum equivalent (von Mises) stress on the root and in the PDL of the first molar

Table 3- Maximum Shear Stress values induced in three models

\begin{tabular}{cccccc}
\hline & Canine & First molar & PDL of Canine & $\begin{array}{c}\text { PDL of First } \\
\text { molar }\end{array}$ & Cortical bone \\
& & & & 0.020853 & 2,0781 \\
\hline Non-surgical & 0.28301 & 0.29064 & 0.028057 & 0.020169 & 0.37052 \\
PD & 0.23029 & 0.22253 & 0.025144 & 0.020165 & 0.62196 \\
\hline
\end{tabular}

$\mathrm{PD}=$ periodontal distraction; $\mathrm{PDL}=$ periodontal ligament $\mathrm{DDO}=$ dentoalveolar distraction osteogenesis

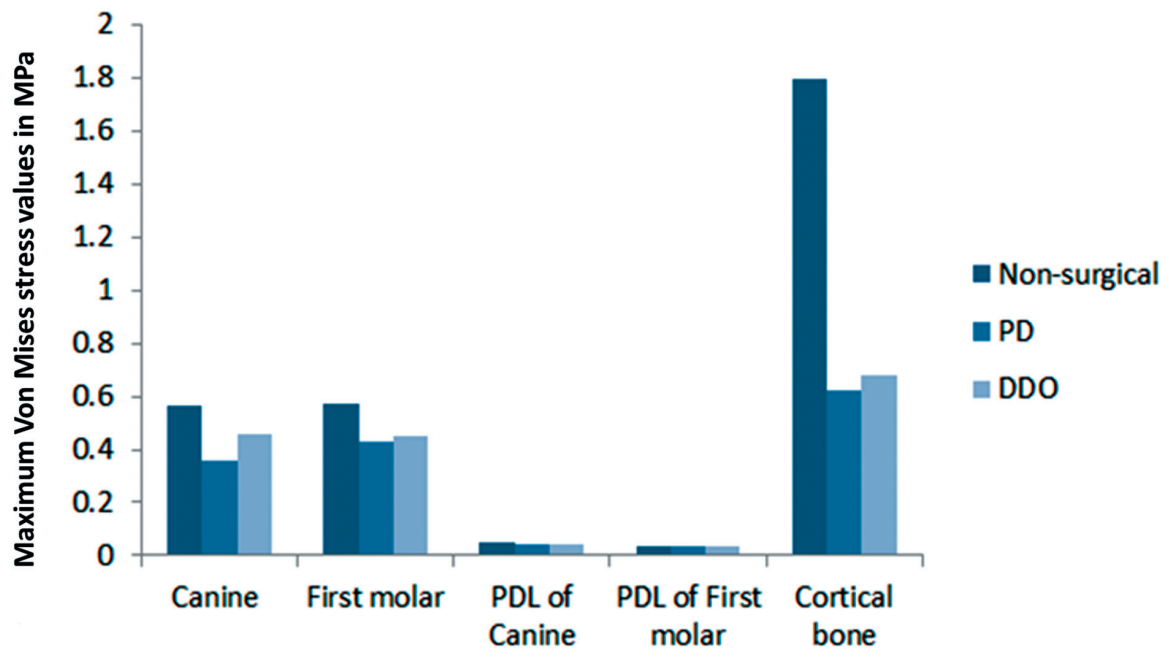

Figure 8- Equivalent (von Mises) stress values induced in different parts of three models. PDL= periodontal ligament

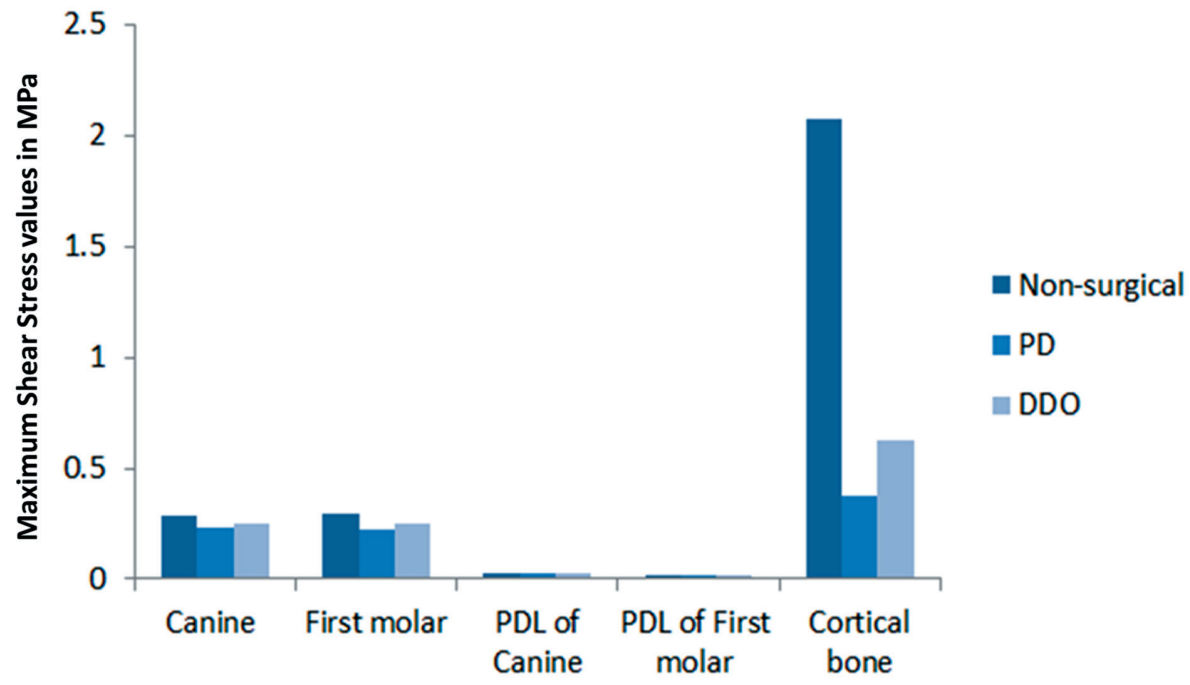

Figure 9- Maximum shear stress values induced in different parts of three models. PDL= periodontal ligament 
in all three models was concentrated in the root furcation of the molar, and the value of maximum equivalent (von Mises) stress of the first molar in model 2 (PD) was the lowest (Table 2). The value and distribution of maximum shear stress on the root and in the PDL of the first molar in all three models showed the same tendency as the maximum equivalent (von Mises) stress, although the values differed.

\section{Alveolar}

Compared with cancellous bone, the buccal side of cortical bone around the canine root was the most stressed area. The value of maximum equivalent (von Mises) stress on the cortical bone around the canine root in model 2 (PD) and model 3 (DDO) was lower than that in model 1 (non-surgical) (Table 2). The value of maximum shear stress on the buccal side of cortical bone around the canine root in model 2 (PD) was the lowest (Table 3).

\section{DISCUSSION}

In this study, orthodontic force was applied to three FEMs which simulated two different surgical interventions and conventional treatment for canine retraction. Stress distribution and deformation on the root, PDL, and cortical bone were evaluated. The stress patterns in the PDL, root, and bone were displayed in separate illustrations, so that the complex responses of these tissues to different types of surgical interventions could be readily compared. The forces were applied to the surfaces of the teeth, mesio-distally, as in normal clinical practice. The results of this study, which showed stress distribution along the root, PDL, and alveolar bone, provided insight into clinical observations.

Orthodontic tooth movement is a biological process characterized by sequential reactions of periodontal tissue against a biomechanical force system $^{6}$. It is also a process in which the application of a mechanical force induces alveolar bone resorption on the pressure side and alveolar bone deposition on the tension side ${ }^{11}$. The orthodontic force system is a complicated three-dimensional system which is difficult to evaluate in clinical conditions and orthodontic force plays an important role in the entire biomechanical process during tooth movement. In our study, it was found that the effects of force application would be changed when the force-loading environment was changed. For accelerating canine retraction speed, periodontal tissue, especially hard tissue such as alveolar bone around the canine, is the most important source of resistance.

Compared with the conventional method of canine retraction, we found that DDO (dentoalveolar distraction osteogenesis) and PD (periodontal distraction aided by surgical undermining of the interseptal bone) could reduce resistance during canine retraction in this finite element analysis. This finding was consistent with those of previous clinical reports ${ }^{10,14}$ and animal experiments ${ }^{7,20}$ with rapid orthodontic tooth movement. Compared with DDO, it was noteworthy, from the mechanical analysis in this study, that PD had more advantages in reducing resistance and accelerating canine movement. It also showed that the stress along the distal side of the canine root was reduced more significantly in model 2 (PD) (Figures 8, 9), with more obvious canine displacement than in model 3 (DDO) (Figure 7 ). The main pathway of canine movement was via the cancellous bone and the interseptal bone, since the main portion of cancellous bone in the pathway of canine distalization was removed by PD. As a result, it is more effective to accelerate canine retraction through periodontal distraction aided by surgical undermining of the interseptal bone.

On the basis of previous studies, resistance to tooth movement is increased when the roots are torqued lingually or buccally. This principle was used by Rickets ${ }^{22}$ (1979) and is called cortical anchorage. Generally, cortical bone offers more resistance to resorption. The cortical bone could also block tooth movement in most cases in orthodontic treatment. In this study, we observed the distribution of stress on the buccal side of cortical bone around the canine root, which implied that the cortical bone on the buccal side of the canine was also the source of resistance to canine movement. That explained the principle of surgical procedures for DDO, which was designed with the dentoalveolus as a bone transport segment for posterior movement ${ }^{10}$. However, based on our results, PD was more effective in canine retraction. Furthermore, in clinical applications, the interseptal bone distal to the canine was undermined to weaken its resistance immediately after dental extraction, and a tooth-borne appliance was used for rapid tooth retraction. This procedure would minimize surgical injury and reduce complications for patients.

In addition to proper orthodontic force, the center of resistance (CR) of a tooth is also a critical factor in predicting and planning the esthetic movement of anterior teeth ${ }^{12}$. Ideally, force should be applied as closely as possible to the CR of a tooth, to achieve movement of the tooth in orthodontic treatment. Although we cannot apply the force on the $C R$, we could change the conditions around the $\mathrm{CR}$. The concentration point of stress on the pressure side of the canine root tended to move closely to the crown after surgical undermining of the interseptal bone in the PD model. In that case, the canine CR became closer to the crown through resistance-reducing surgery, especially in the PD model, and this would lead to possible canine 
body movement. Otherwise, the lowest maximum equivalent (von Mises) stress value on the distal side of the root surface in model 2 (PD) implied that reducing the resistance around the canine $C R$ in that model would reduce the stress value. The lower and more uniform stress on the root indicated that translational tooth movement may be achieved, which made it more effective in accelerating canine retraction speed.

However, it should be pointed out that the concentrated point of force loading at the distal side of the canine cervix for the PDL and alveolar bone shifted to the lingual side in the PD model, and, conversely, in the DDO model, this point shifted to the buccal side. This indicated to us that the canine had a tendency to rotate lingually in the PD model and buccally in the DDO model after resistance was reduced. The reason for this might be that after the different positions of resistance source were reduced by surgery, the canine would move to the position with the lowest resistance. Therefore, we should avoid this side-effect when using PD or DDO during orthodontic treatment.

Anchorage control should be considered when accelerated tooth movement is necessary. An obvious strategy for anchorage control would be to concentrate the force needed to produce tooth movement where it was desired, and then to dissipate the retraction force in the PDL of anchor teeth as much as possible. In this study, the value of maximum equivalent (von Mises) stress in the PDL of the first molar in two models was surgical reduction of resistance to a level lower than in the canine. Further, the value of total deformation of the first molar in the DDO and PD models was far below that of the canine at the initial stage of force loading. This indicated that reducing resistance by DDO or PD to accelerate canine retraction was a safe way to protect anchorage and would not reduce anchorage during canine retraction. Furthermore, the pattern of force distribution in the PDL and root of the first molar showed that high stress concentration is observed on the root surface at the furcation level, which is in contrast to the canine, which displayed a greater apical concentration. This may explain the high incidence of apical root resorption ${ }^{25}$ that appears to occur in the maxillary molar on the root surface at the furcation level, which is difficult to see radiographically in orthodontic treatment.

This study and others have demonstrated that FEM provides a solid, workable foundation for modeling a system of orthodontic tooth movement ${ }^{1}$. The chief advantage of FEM is that it can be magnified nearly infinitely, in terms of both the actual volumetric construction itself and the mathematical variability of its material parameters. However, as with any theoretical model of a biological system, there are limitations. The mechanical behavior of the materials was assumed to be linear elastic (homogeneous and isotropic), and the value of each material was inferred from previous reports. Cortical bone thickness and cancellous bone quality were not incorporated into the analysis, to prevent bone stress from being dominated by bone quality and potentially confounding the outcomes related to other relevant factors. In addition, the stress analysis of soft tissues was not considered in this study. The soft tissues, such as gingival and facial muscles, are also sources of resistance for blocking rapid tooth movement. Regardless of these limitations, we integrated a finite element approach with variable analysis to investigate the comparative influences of resistance source, the pathway of canine movement, and different types of surgeries for rapid canine retraction by reducing resistance.

\section{CONCLUSIONS}

In this study, compared with dento-alveolar distraction osteogenesis, periodontal distraction aided by surgical undermining of the interseptal bone would reduce the resistance in the pathway of canine movement more effectively to accelerate canine retraction speed in rapid canine movement during orthodontic treatment.

The results indicated that rapid canine retraction aided by the surgical reduction of resistance might create side effect: 1 ) it might lead to canine rotation during distalization, 2) the geometry of the root of maxillary first molar makes it less prone to apical stress concentration, therefore, resorption on the root surface at the furcation level of the molars is more easily caused.

\section{ACKNOWLEDGEMENTS}

This study was financially supported by the National Natural Science Foundation of China (General Program) (Grant No. 81070858), the National Natural Science Foundation of China for Young Scholars (Grant No. 81100778), and the Natural Science Foundation of Xinjiang province of China for Young Scholars (Grant No. 2011211B35).

\section{REFERENCES}

1- Archangelo CM, Rocha EP, Pereira JA, Martin Junior M, Anchieta RB, Freitas Júnior AC. Periodontal ligament influence on the stress distribution in a removable partial denture supported by implant: a finite element analysis. J Appl Oral Sci. 2012;20:362-8.

2- Codivilla A. The classic: On the means of lengthening, in the lower limbs, the muscles and tissues which are shortened through deformity. 1905. Clin Orthop Relat Res. 2008;466:2903-9.

3- Cuoghi OA, Tondelli PM, Aiello CA, Mendonca MR, Costa SC. Importance of periodontal ligament thickness. Braz Oral Res. 2013;27:76-9.

4- Fisher MA, Wenger RM, Hans MG. Pretreatment characteristics associated with orthodontic treatment duration. Am J Orthod Dentofacial Orthop. 2010;137:178-86. 
5- Geiger AM, Gorelick L, Gwinnett AJ, Benson BJ. Reducing white spot lesions in orthodontic populations with fluoride rinsing. Am J Orthod Dentofacial Orthop. 1992;101:403-7.

6- Geramy A. Alveolar bone resorption and the center of resistance modification (3-D analysis by means of the finite element method). Am J Orthod Dentofacial Orthop. 2000;117:399-405.

7- Iino S, Sakoda S, Ito G, Nishimori T, Ikeda T, Miyawaki

S. Acceleration of orthodontic tooth movement by alveolar corticotomy in the dog. Am J Orthod Dentofacial Orthop. 2007; 131:448.e1-8.

8- Işeri $H$, Kişnişci R, Bzizi N, Tüz $H$. Rapid canine retraction and orthodontic treatment with dentoalveolar distraction osteogenesis. Am J Orthod Dentofacial Orthop. 2005;127:533-51,625.

9- Kawakami M, Takano-Yamamoto T. Local injection of 1,25-dihydroxyvitamin D3 enhanced bone formation for tooth stabilization after experimental tooth movement in rats. J Bone Miner Metab. 2004;22:541-546.

10- Kharkar VR, Kotrashetti SM. Transport dentoalveolar distraction osteogenesis-assisted rapid orthodontic canine retraction. Oral Surg Oral Med Oral Pathol Oral Radiol Endod. 2010;109:687-93. 11- Kiliç N, Oktay $H$, Ersöz M. Effects of force magnitude on tooth movement: an experimental study in rabbits. Eur J Orthod. 2010;32:154-8.

12- Kusy RP, Tulloch JF. Analysis of moment/force ratios in the mechanics of tooth movement. Am J Orthod Dentofacial Orthop. 1986;90:127-31.

13- Lim JE, Lee SJ, Kim YJ, Lim WH, Chun YS. Comparison of cortical bone thickness and root proximity at maxillary and mandibular interradicular sites for orthodontic mini-implant placement. Orthod Craniofac Res. 2009;12:299-304.

14- Liou EJ, Huang CS. Rapid canine retraction through distraction of the periodontal ligament. Am J Orthod Dentofacial Orthop. $1998 ; 114: 372-82$.

15- Marquezan M, Bolognese AM, Araújo MT. Evaluation of two protocols for low-level laser application in patients submitted to orthodontic treatment. Dental Press J Orthod. 2013;18:33.e1-9. 16- McCarthy JG, Schreiber J, Karp N, Thorne CH, Grayson BH. Lengthening the human mandible by gradual distraction. Plast Reconstr Surg. 1992;89:1-8,9-10.
17- Ozdemir F, Tozlu M, Germec-Cakan D. Cortical bone thickness of the alveolar process measured with cone-beam computed tomography in patients with different facial types. Am J Orthod Dentofacial Orthop. 2013;143:190-6.

18- Pandis N, Nasika M, Polychronopoulou A, Eliades T. External apical root resorption in patients treated with conventional and self-ligating brackets. Am J Orthod Dentofacial Orthop. 2008; $134: 646-51$.

19- Reimann S, Keilig L, Jager A, Bourauel C. Biomechanical finiteelement investigation of the position of the centre of resistance of the upper incisors. Eur J Orthod. 2007;29:219-24.

20- Ren A, Lv T, Kang N, Zhao B, Chen Y, Bai D. Rapid orthodontic tooth movement aided by alveolar surgery in beagles. Am J Orthod Dentofacial Orthop. 2007;131:160-1.

21- Richardson ER. Comparative thickness of the human periodontal membrane of functioning versus non-functioning teeth. J Oral Med. 1967;22:120-6.

22- Ricketts RM. Bioprogressive therapy. Denver: Rocky Mountain/ Orthodontics; 1979.

23- Rygh P. Elimination of hyalinized periodontal tissues associated with orthodontic tooth movement. Scand J Dent Res. 1974;82:5773.

24- Vásquez M, Calao E, Becerra F, Ossa J, Enríquez C, Fresneda E. Initial stress differences between sliding and sectional mechanics with an endosseous implant as anchorage: a 3-dimensional finite element analysis. Angle Orthod. 2001;71:247-56.

25- Vieira-Andrade RG, Drumond CL, Alves LP, Marques LS, Ramos-Jorge ML. Inflammatory root resorption in primary molars: prevalence and associated factors. Braz Oral Res. 2012;26:335-40. 26- Vig PS, Weintraub JA, Brown C, Kowalski CJ. The duration of orthodontic treatment with and without extractions: a pilot study of five selected practices. Am J Orthod Dentofacial Orthop. 1990;97:45-51. 